\title{
Collective radiation dose from diagnostic x-ray examination in nine public hospitals in Addis Ababa, Ethiopia
}

\author{
Daniel Admassie ${ }^{1}$, Seife Teferi ${ }^{1}$, Kalkidan Hailegenaw $^{1}$
}

\begin{abstract}
Background: Medical x-ray exposures have the largest man made source of population exposure to ionizing radiation in different countries. Recent developments in medical imaging have led to rapid increases in a number of high dose xray examinations performed with significant consequences for individual patient doses and for collective dose to the population as a whole. It is therefore important in each country to make regular assessments of the magnitude of these large doses.

Objectives: To calculate collective dose of the population as a result of radiation dose from diagnostic $\mathrm{x}$-rays, thereby to estimate the annual incidence of cancer which would be reduced by the use of rare earth intensifying screen.

Methods: Data on the number of diagnostic procedures using x-ray examination in year 2007 in nine governmental hospitals, excluding military hospitals, by body site were collected in Addis Ababa. The number of examinations of specific body site was multiplied by the average effective dose per examination to get the collective dose over the population. Based on International Commission on Radiological Protection (ICRP) the fatality risk of fatal cancers (5\% per Sv) was estimated.

Results: In this study, the annual collective dose over the population is $31.21 \mathrm{manSv}(0.0 .42 \mathrm{mSv}$ per person). Based on ICRP fatality risk of 500 fatal cancers per 10,000 man-sieverts (5\% per Sv), estimation of incidence of fatal cancers cases in year 2007 was 2 cases half of which can be reduced by adoption of rare earth screens.

Conclusion: Although the use of ionizing radiation for diagnostic medical procedures is an acceptable part of modern medicine, there is also the potential for inappropriate use and unnecessary radiation dose to the patient, so the request of radiography must be justified. It is estimated that the adoption of rare earth screen technology might reduce the annual incidence of cancer which would be fatal after an average latency period of 18.4 years by half, hence this research recommended adopting rare earth screen technology in Ethiopia. [Ethiop. J. Health Dev. 2010;24(2):140-
\end{abstract} 144]

\section{Introduction \\ Intensifying screens in general consist of a thin layer of tiny phosphor crystal mixed with a suitable binder and coated in a smooth layer on a plastic support or card board. The basic principle in the action of intensifying screen is utilization of a phosphor that converts energy carried by an x-ray photon into visible light.}

The purpose of these screens is to reduce radiation exposure required to produce a diagnostic radiograph. This results in the usage of lower mAs (milli-amperesecond) setting which is advantageous because of the ability to utilize shorter exposure times. In addition, patient dosage is reduced, motion non-sharpness will not be a serious problem and film contrast is improved through the use of intensifying screens $(1,2)$.

The speed at which intensifying screens can convert $\mathrm{x}$ ray energy to radiation depends on physical properties (i.e. size and number of phosphors) and type of phosphor which is critical because of the ability to absorb x-ray energy. The conventional intensifying screens have low absorption coefficient and conversion efficiency as compared to newly developed rare earth screens $(1,2)$.
Rare earth intensifying screens are derived from rare earth elements of lanthanide series. Their noteworthy character is their usually high $\mathrm{x}$-ray absorption coefficient and high $\mathrm{x}^{-}$ray to light conversion effectiveness (2).

Newlin described that the cost of rare earth screens is about double that of calcium tungstate screens and the cost may be offset by the increase in tube life (1). It is estimated also that $50 \%$ decrease in the cumulative radiation dose is related to the cumulative radiation quantity emitted by the tube, which will increase the tube life (3).

The carcinogenic effect of sub lethal radiation doses was regarded as a major population risk when excess leukemia began to appear in the Japanese atomic bomb survivors in the late forties.

The decrease in radiation dose of patients undergoing diagnostic $\mathrm{x}$-ray has a significant value. The United Nations Scientific Committee on the Effects of Atomic Radiation, in its 1972 report, says that the protection of the patient is probably the greatest factor in control of population exposures. The aim is not only to reduce the radiation exposure of individuals but also to have

${ }^{1}$ Department of Radiology, Medical Faculty, Addis Ababa, University, Tel. 0911229890, e-mail

dadmasie@gmail.com or seifeteferi@yahoo.com, Tel. 0911648916 
procedures carried out with maximum efficiency so that there can be continuing increase in medical benefits accompanied by a minimum radiation exposure. This can also be achieved by the use of rare earth screen $(1,2)$.

Diagnostic x-rays are the largest man made sources of radiation exposure to the population, contributing to about $14 \%$ of the total annual exposure worldwide from all sources. Although diagnostic $\mathrm{x}$-ray provides great benefits, its use carries some risk of developing cancer (4, $5)$.

Medical practice is the biggest man made source of radiation. Justified examination and treatments contribute greatly to health as welfare, but side effects include a risk of cancer and other stochastic effects in proportion to the dose (6). But this dose and incidence of cancer can be reduced by the use of rare earth intensifying screen (3). Ethiopia is therefore required to implement the use of rare earth screen technology due to the above mentioned benefits.

In a study done at the University of Michigan student health service, two screen film combinations were compared with rare earth screen and calcium tungestate screens. The films were processed under the same condition. The result showed that exposure time was significantly reduced using the rare earth screen/film combination (1).

Another study on plain radiography was performed on 388 patients using calcium tungestate and rare earth screen. Radiographs were compared for overall image quality, mottle, contrast, density and detail. The study showed that the rare earth screens produce radiographs with more mottle than did the standard screen. It also showed that the major advantage of rare earth screens is a $50 \%$ or more reduction in radiation exposure which is a benefit outweighing a small and clinically insignificant decrease in overall image quality (2).

It is claimed that of all approaches with clear impact on dose reduction, the replacement of the conventional image intensifying screens in $\mathrm{x}$-ray cassette with the rare earth screens has been shown to reduce patient dose to half or more, still in a very cost effective manner $(3,7,8)$.

This research is therefore conducted to calculate collective dose of the population as a result of radiation dose from diagnostic x-rays and to estimate the annual incidence of cancer which would be reduced by the use of rare earth intensifying screen.

\section{Methods}

Data were collected on the number of diagnostic procedures using x-ray examination in the year 2007 in nine public hospitals by body site, in the Ethiopian capital Addis Ababa.
The Hospitals included in the study are Menilik II, Yekatit 12, Saint Paul, Gandhi Memorial, Ras Desta, Zewditu Memorial, Tikur Anbesa, ALERT and Saint Peter. These hospitals were chosen for the study because they are the largest hospitals in the country in terms of workload. The study was retrospective and included all diagnostic x-ray examinations in year 2007. The data were entered into a table registering the number of $x$-ray examinations in the specified period by body site.

The examinations included in the data were plain radiographs which were categorized into 9 body sites. All intravenous urography (IVU) studies and barium examination of the gastrointestinal tract (GIT) series in the specified one year period were recorded. The number of examinations helps to calculate the collective effective dose.

Collective effective dose is the quantity obtained by multiplying the average effective dose by the number of people exposed to a given source of ionizing radiation. The Systemic International (SI) unit is man Sievert (manSv). Effective dose is the sum of doses to each organ in the irradiated volume weighted according to the radio sensitivity of each organ. Its SI unit is millisievert (msv) (9-11).

In the case where a country is not able to make extensive patient dose measurements and to estimate nationally representative effective doses for all types of $\mathrm{x}$-ray examinations, it is usual practice to use published values from the literature $(12,13)$. Patient doses for the same examination are known to vary widely between countries and even between hospitals in the same country, so estimates of national mean doses based on just local or foreign data will not be very reliable (12). However, for those countries currently without the resources to make extensive national patient dose surveys, some sets of 'typical' effective doses for those examinations making major contributions to collective dose are provided by European Commission (12).

Hence, since there is no study done in Ethiopia to estimate the average effective dose per examination, in this research the average effective dose per examination per body site was taken from suggested values by European commission (12).

An estimate of the number of fatal cancers that may be caused by exposure to diagnostic $x$-ray radiation was made using the International Commission on Radiological Protection Report (ICRP) of 500 fatal cancers per 10,000 manSv given to the standard population $(5 \%$ per Sv $)(3,14)$.

\section{Results}

The total number of diagnostic $\mathrm{x}$ ray examinations in the 9 hospitals was 89,354 in year 2007. The examinations included in the study were all plain radiographs, IVU and barium contrast studies of the GIT.

Ethiop. J. Health Dev. 2010;24(2) 
In Tikur Anbesa Hospital, which is the largest and main referral hospital, the number of diagnostic x-ray examinations was the highest with 39,343 (Fig 1). In addition to this, in Tikur Anbesa Hospital, all the various diagnostic examinations included in the study were performed. Menilik II and Zewditu Memorial hospitals conducted the next highest number of diagnostic x-ray examinations. The least number of diagnostic examinations were done in Gandhi Memorial hospital followed by ALERT (Fig 1). IVU and barium contrast GIT studies were done in only 3 of the hospitals (Tikur Anbesa, Menilik II and Zewditu Memorial hospitals).

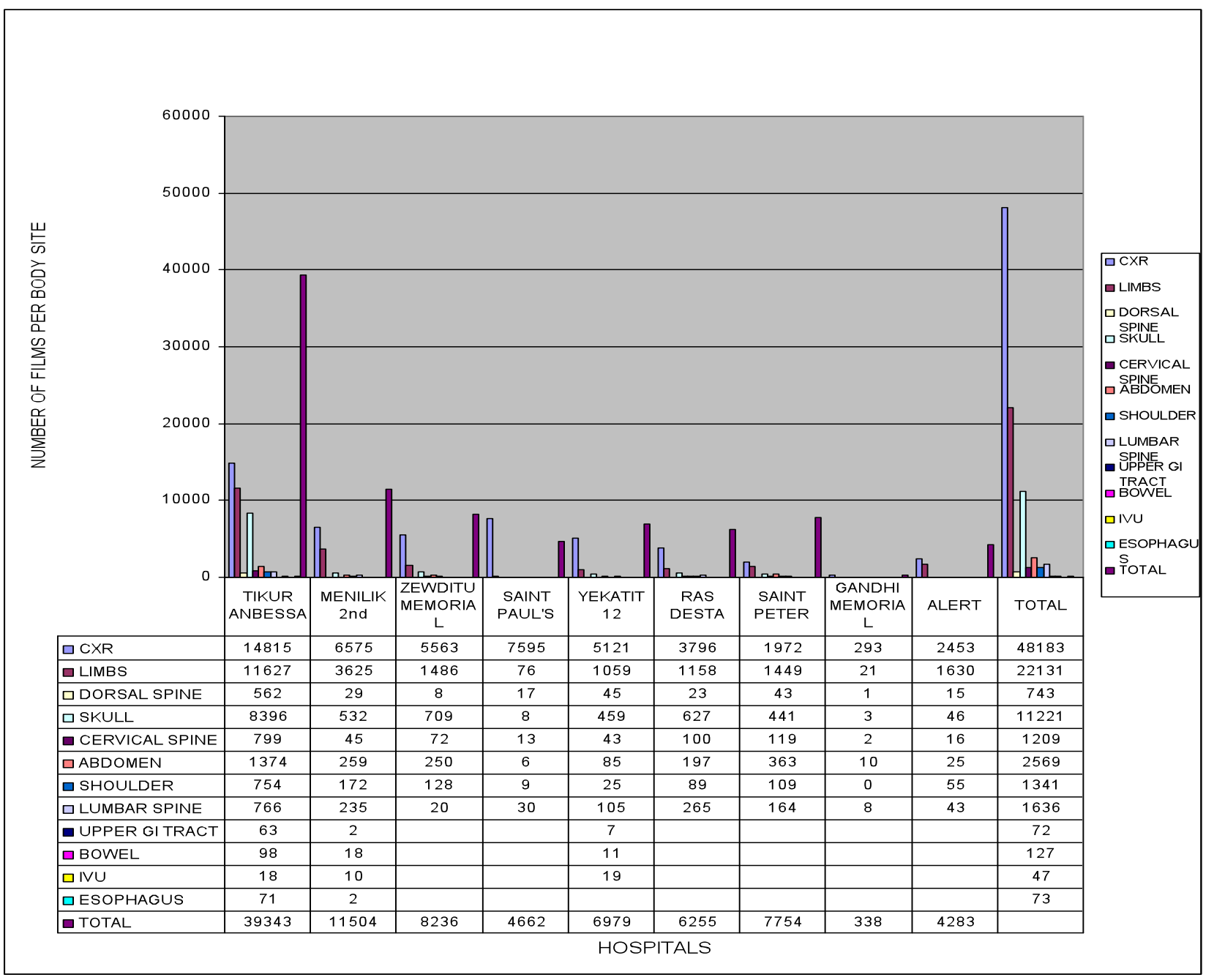

Figure 1: Diagnostic x-ray examination in Addis Ababa in 2007 from nine governmental hospitals.

In year 2007, out of a total of 89,354 radiography films taken in nine hospitals in Addis Ababa, chest and limbs accounted for $54 \%$ and $24.7 \%$ respectively. They only accounted for $38.6 \%$ and $7.1 \%$ respectively of the total annual effective dose received due to their low effective dose per examination (Table 1).

Plain abdominal and lumbar spine films accounted for $14.8 \%$ and $14.6 \%$ of the total annual effective doses received followed by dorsal spine which accounted for $4.7 \%$. Barium tests, which were performed in only three hospitals, accounted for $0.33 \%$ of all examinations and for $12.6 \%$ of the total effective dose, which is significant due to their relatively higher effective dose per examination (Table 1).

The annual total collective radiation dose received by the population in nine public hospitals in Addis Ababa from film radiography that could be possibly reduced by the use of rare earth screens in 2007 was 31.21 manSv (Table 1). Based on ICRP (International Commission on Radiological Protection) fatality risk of 500 fatal cancers per 10,000 man-SV (5\% per Sv), estimation of incidence of fatal cancer cases in year 2007 was 2, half of which could be reduced by adoption of rare earth screens. 


\begin{tabular}{|c|c|c|c|c|c|}
\hline Body site & $\begin{array}{l}\text { Effective dose } \\
\text { (mSv) per exam }\end{array}$ & $\begin{array}{l}\text { Number of } \\
\text { examination }\end{array}$ & $\begin{array}{l}\% \text { of } \\
\text { examination out } \\
\text { of total } \\
\text { examination }\end{array}$ & $\begin{array}{l}\text { Total collective } \\
\text { Effective dose } \\
\text { (man-Sv) }\end{array}$ & $\begin{array}{l}\% \text { of collective } \\
\text { effective dose } \\
\text { Out of total } \\
\text { collective effective } \\
\text { dose }\end{array}$ \\
\hline Chest X-ray & 0.25 & 48183 & 54 & 12.04575 & 38.61 \\
\hline Limbs & 0.1 & 22131 & 24.7 & 2.2131 & 7.09 \\
\hline Shoulder & 2.8 & 1341 & 1.5 & 0.33525 & 1.07 \\
\hline Lumbar spine & 0.1 & 1636 & 1.8 & 4.5808 & 14.68 \\
\hline Skull & 0.7 & 11221 & 12.5 & 1.1221 & 3.60 \\
\hline Cervical spine & 2 & 1209 & 1.4 & 0.8463 & 2.71 \\
\hline Dorsal spine & 1.8 & 743 & 0.8 & 1.486 & 4.76 \\
\hline Abdomen & 15 & 2571 & 2.9 & 4.6278 & 14.83 \\
\hline Upper GI & 12.5 & 72 & 0.08 & 1.08 & 3.46 \\
\hline Bowel & 4 & 127 & 0.14 & 1.5875 & 5.09 \\
\hline IVP & 15 & 47 & 0.05 & 0.188 & 0.60 \\
\hline Esophagus & & 73 & 0.08 & 1.095 & 3.51 \\
\hline Total & & 89354 & 100 & 31.21 & 100 \\
\hline
\end{tabular}

\section{Discussion}

One of the ways to achieve the largest reduction in radiation exposure may be to exclude the prescription of unnecessary or unproductive x-ray examinations. Patient exposure can also be reduced by assuring that good radiographic technique is practiced. It was said that the fundamental objective of x-ray examination is to obtain optimum diagnostic information with minimum diagnostic exposure. This can be achieved by the use of rare earth screens. Rare earth intensifying screen gives shorter exposure time without clinically significant decrease in image quality $(1,2,7)$.

The annual collective radiation dose received from diagnostic radiology in year 2007 in nine public Hospitals in Addis Ababa was $31.21 \mathrm{manSv}(0.42 \mathrm{mSv} /$ person). The average radiation dose received per person $(0.42 \mathrm{mSv} /$ person $)$ is less than that of the average radiation dose received per person for the Israeli populations $(0.53 \mathrm{mSv} /$ person) (3) and higher than Iranian population $(0.03 \mathrm{mSv} /$ person) (14). This is explained by the fact that, out of a total of 89,354 radiography films taken in Addis Ababa, chest which has lowest effective dose accounted for $54 \%$ of examination $(38.61 \%$ of collective dose). Further, highly effective dose contributors like bowel (Barium studies) and dorsal spine are not performed in wide scale in Addis Ababa as compared for example to Israel (3).

Based on the fatality risk of $5 \%$ per sievert (3) we estimate that in nine public Hospitals in Addis Ababa approximately 2 fatal cases of cancer $(31.21 \mathrm{manSv} \times 5 \%$ per sievert) may in the future be attributable to diagnostic x-rays done in year 2007. The adoption of rare earth intensifying screens would have resulted in a $50 \%$ reduction in radiation dosage from diagnostic x-rays (1, 4). Since the average latency period of mortality from diagnostic $\mathrm{x}$-rays is 18.4 years (11), the number of cases of fatal cancers attributable to diagnostic $\mathrm{x}$-rays after 18.4 years of latency period in public hospitals in Addis
Ababa would be reduced to half if rare earth screens are used.

Estimation of the extent of this risk on the basis of the annual number of diagnostic x-rays undertaken in the UK and 14 other developed countries were done. The result indicates that in the UK about $0.6 \%$ of the cumulative risk of cancer to age 75 years could be attributable to diagnostic x-rays (4). This percentage is equivalent to about 700 cases of cancer per year. In other 13 developed countries estimates of the attributable risk ranged from 0.6 to $1.8 \%$, whereas it was more than $3 \%$ in Japan, which had the highest estimated annual exposure frequency in the world (4).

The adoption of rare earth screen provides an effective means to prevent ionizing radiation exposure to the population due to the valuable radiation reduction if used in the place of the conventional intensifying screen and also saves the cost of life time treatment of cancer $(1,2$, $3)$.

The reduction in radiation dose not only results in reduction in radiation associated cancer but also prolongs the x-ray tube life. This is due to the fact that rare earth intensifying screens are faster than conventional screens as a consequence of shorter exposure times. This dose reduction is related to the cumulative radiation quality emitted by the tube which will increase the tube life by around $60 \%(3)$.

Generally, the cost of rare earth intensifying screens is estimated to be double that of conventional screens $(1,3)$. The cost benefit of cancer risk reduction associated with diagnostic $x$-radiation with specific cost can not be estimated in case of our country because there is no data on life time treatment cost of cancer.

Even though many developing countries such as Ethiopia are switching to digital computed radiography, it can be 
used as a good transition to use rare earth screen in a cost effective manner.

Finally, we recommend that the findings of the present work can be used as a baseline upon which a large scale study including all hospitals in Ethiopia should be done.

Although the use of ionizing radiation for diagnostic medical procedures is an acceptable part of modern medicine, there is also the potential for inappropriate use and unnecessary exposure of patients to radiation doses. Therefore, each request for radiography must be justified. Since the dose and incidence of cancer can be reduced by the use of rare earth intensifying screen, this research article recommends adopting rare earth screen technology in Ethiopia.

\section{References}

1. Newlin N. Reduction in radiation exposure: the rare earth screen. AJR 1978;130:1195-1196.

2. Daniel P. Plain radiography with rare earth screen, comparison with calcium tungstate screen. AJR 1984;143:1335-1338.

3. Ginsberg GM, Schlesinger T, Ben Shomo A. An economic evaluation of use of rare earth screens to reduce the radiation doses from diagnostic $\mathrm{x}$-ray procedures in Israel. BJR 1998;71:406-412.

4. de González A, Darby S. Risk of cancer from diagnostic X-rays: estimates for the UK and 14 other countries. The Lancet 2004;363(9406):345- 351.

5. Health Risks of Low Level Radiation Exposure. Indian Journal of Nuclear medicine 2005;20(2):

6. Effective doses to members of the public from diagnostic application of ionizing radiation in Germany. European Radiology Journal 1997;7(7):
7. Madan R, Pedero L. Protection of patients in medical exposure, International Atomic Energy Agency, Vienna, Austria.

8. Engel-Hills P. Radiation protection in medical imaging Radiography 2006; 12(2):153-160.

9. Cynthia H, Beth A. Calculation of Effective Dose: Educational Treatise. Medical Physics 2000;27(5): 828-837.

10. Brenner D, Hunde W. Effective Dose, a useful concept in diagnostic radiology. Radiation Protection Dosimetery 2008;128(4):503-504.

11. Tyndall DA, Washburn DB. The effect of rare earth filtration on patient exposure, dose reduction, and image quality in oral panoramic radiology. Health Physics 1987; 52(1):17-26.

12. European Commission. European Guidance on Estimating Population Doses from Medical X-ray Procedures. Radiation Protection 2008;154.

13. International Basic Safety Standards for Protection Against Ionizing Radiation and for the Safety of Radiation Sources, Safety Series No. 115 IAEA 1996; Jointly sponsored by FAO, ILO,OECD/NEAPAHO and WHO.

14. International Commission on Radiological Protection. Recommendation of the International commission on Radiological Protection. (ICRP Publication 60) Ann ICRP 1991;21:13.

15. Bouarjomehri F, Dashti M, Zare M. Radiation exposure of the Yazd population from medical conventional X-ray examinations. Iran. J Radiat Res 2007;4(4):195-200. 